УДК 517.9

\title{
On a Second Order Linear Parabolic Equation with Variable Coefficients in a Non-Regular Domain of $\mathbb{R}^{3}$
}

\author{
Ferroudj Boulkouane* \\ Faculté des science de la nature et de la vie \\ Université de Bejaia, 6000 Béjaia \\ Algérie
}

Arezki Kheloufi

Faculty of Technology, Lab. of Applied Mathematics

Bejaia University, 6000 Béjaia

Algéria

Received 11.10.2017, received in revised form 22.01.2018, accepted 06.03.2018

This paper is devoted to the study of the following variable-coefficient parabolic equation in non-divergence form

$$
\partial_{t} u-\sum_{i=1}^{2} a_{i}\left(t, x_{1}, x_{2}\right) \partial_{i i} u+\sum_{i=1}^{2} b_{i}\left(t, x_{1}, x_{2}\right) \partial_{i} u+c\left(t, x_{1}, x_{2}\right) u=f\left(t, x_{1}, x_{2}\right)
$$

subject to Cauchy-Dirichlet boundary conditions. The problem is set in a non-regular domain of the form

$$
\left.Q=\left\{\left(t, x_{1}\right) \in \mathbb{R}^{2}: 0<t<T, \varphi_{1}(t)<x_{1}<\varphi_{2}(t)\right\} \times\right] 0, b[,
$$

where $\varphi_{k}, k=1,2$ are "smooth" functions. One of the main issues of this work is that the domain can possibly be non-regular, for instance, the singular case where $\varphi_{1}$ coincides with $\varphi_{2}$ for $t=0$ is allowed. The analysis is performed in the framework of anisotropic Sobolev spaces by using the domain decomposition method. This work is an extension of the constant-coefficients case studied in [15].

Keywords: parabolic equations, non-regular domains, variable coefficients, anisotropic Sobolev spaces. DOI: 10.17516/1997-1397-2018-11-4-416-429.

\section{Introduction and main results}

This work is devoted to the study of the following two-space dimensional non-divergence parabolic equation

$$
\left\{\begin{array}{l}
\partial_{t} u+\mathcal{L} u=f \in L^{2}(Q), \\
\left.1 u\right|_{\partial Q \backslash \Sigma_{T}}=0
\end{array}\right.
$$

where

$$
\mathcal{L}=-\sum_{i=1}^{2} a_{i}\left(t, x_{1}, x_{2}\right) \partial_{i i}+\sum_{i=1}^{2} b_{i}\left(t, x_{1}, x_{2}\right) \partial_{i}+c\left(t, x_{1}, x_{2}\right)
$$

with $\partial_{i}=\frac{\partial}{\partial x_{i}}, \partial_{i i}=\frac{\partial^{2}}{\partial x_{i}^{2}}, i=1,2 . L^{2}(Q)$ stands for the space of square-integrable functions on $Q$ with the measure $d t d x_{1} d x_{2}, \partial Q$ is the boundary of $Q, \Sigma_{T}$ is the part of the boundary of $Q$ where $t=T$ and the coefficients $a_{i}, b_{i}, i=1,2$ and $c$ satisfy non-degeneracy-assumptions (to be made more precise later). Here $Q$ (see, Fig. 1) is the three-dimensional non-cylindrical domain

$$
\left.Q=\left\{\left(t, x_{1}\right) \in \mathbb{R}^{2}: 0<t<T, \varphi_{1}(t)<x_{1}<\varphi_{2}(t)\right\} \times\right] 0, b[,
$$

* ferroudjboulkouane@gmail.com

(c) Siberian Federal University. All rights reserved 
where $T$ and $b$ are positive numbers, $\varphi_{1}$ and $\varphi_{2}$ are two Lipschitz continuous real-valued functions on $[0, T]$ satisfying

$$
\left.\left.\varphi(t):=\varphi_{2}(t)-\varphi_{1}(t)>0, \forall t \in\right] 0, T\right] \text { and } \varphi(0)=0 .
$$

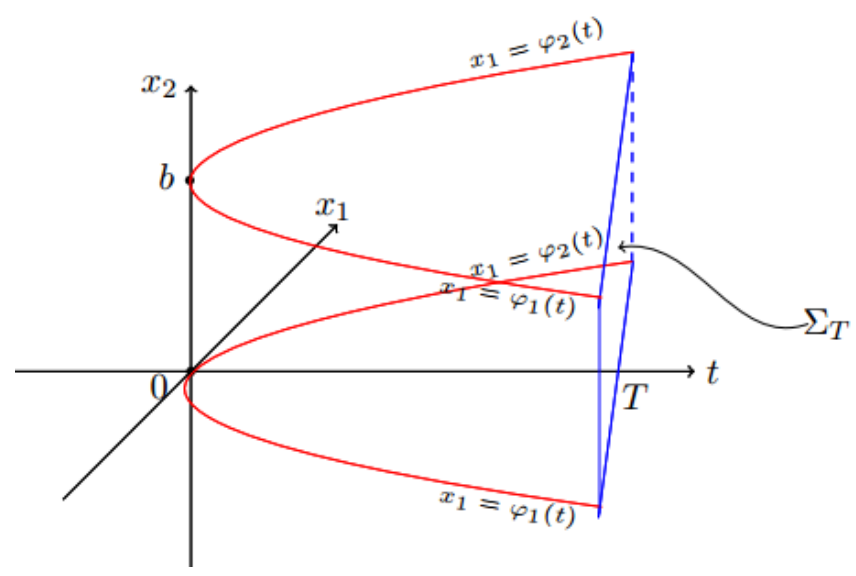

Fig. 1. The non-regular domain $Q$

Besides being interesting in itself, Problem (1.1) governs, for instance, the concentration of the biological oxygen demand in water in the case of a river with variable width and constant depht, see for example, similar problems in [1] and [31]. Also, the particular form of the operator $\mathcal{L}$ helps us to prove the "energy" type estimate of Proposition 2.1 which is essential in proving the existence of solutions to Problem (1.1).

The difficulty related to this kind of problems (in addition to the presence of variable coefficients) comes from this singular situation for evolution problems, i.e., $\varphi_{1}$ is allowed to coincide with $\varphi_{2}$ for $t=0$, which prevents the domain $Q$ to be transformed into a regular domain without the appearance of some degenerate terms in the parabolic equation, see for example Sadallah [30]. On the other hand, we cannot recast such problems in semigroups setting like in [6] and [27]. Indeed, since the initial condition is defined on a measure zero set, then the semigroup generating the solution cannot be defined.

It is well known that there are two main approaches for the study of boundary value problems in such non-smooth domains. We can work directly in the non-regular domains and we obtain singular solutions (see, for example $[3,16,18]$ and $[20]$ ), or we impose conditions on the non-regular domains (and on the coefficients) to obtain regular solutions (see, for example $[2,17]$ and [30]). It is the second approach that we follow in this work. So, let us consider the anisotropic Sobolev space

with

$$
\mathcal{H}_{0}^{1,2}(Q)=\left\{u \in \mathcal{H}^{1,2}(Q):\left.u\right|_{\partial Q \backslash \Sigma_{T}}=0\right\}
$$

$$
\mathcal{H}^{1,2}(Q)=\left\{u: \partial_{t} u, \partial^{\alpha} u \in L^{2}(Q),|\alpha| \leqslant 2\right\},
$$

where

$$
\alpha=\left(i_{1}, i_{2}\right) \in \mathbb{N}^{2},|\alpha|=i_{1}+i_{2}, \partial^{\alpha} u=\partial_{1}^{i_{1}} \partial_{2}^{i_{2}} u .
$$

The space $\mathcal{H}^{1,2}(Q)$ is equipped with the natural norm, that is

$$
\|u\|_{\mathcal{H}^{1,2}(Q)}=\left(\left\|\partial_{t} u\right\|_{L^{2}(Q)}^{2}+\sum_{|\alpha| \leqslant 2}\left\|\partial^{\alpha} u\right\|_{L^{2}(Q)}^{2}\right)^{1 / 2} .
$$


In this paper we prove that Problem (1.1) admits a unique solution $u$ in $\mathcal{H}^{1,2}(Q)$, under the following additional assumptions on the smooth differentiable coefficients $c, a_{i}, b_{i}, i=1,2$ and on the functions of parametrization $\varphi_{k}, k=1,2$,

$$
\begin{gathered}
\varphi_{k}^{\prime}(t) \varphi(t) \rightarrow 0 \text { as } t \rightarrow 0, \quad k=1,2, \\
\left\{\begin{array}{l}
a_{i}>0 \text { (parabolicity condition) } \\
a_{i}, b_{i}, c, \partial_{t} a_{i}, \partial_{i} a_{i} \in L^{\infty}(Q), i=1,2,
\end{array}\right.
\end{gathered}
$$

with $\left|a_{i}\right| \leqslant c_{0},\left|\nabla a_{i}\right| \leqslant c_{1},\left|b_{i}\right| \leqslant c_{2},|c| \leqslant c_{3}, a_{i} a_{j} \geqslant a_{0}>0(i ; j=1,2), b_{i}^{2} \geqslant b_{0}>0, c^{2} \geqslant d_{0}>0$, where $c_{0}, c_{1}, c_{2}, c_{3}, a_{0}, b_{0}$ and $d_{0}$ are positive constants.

Our main result is

Theorem 1.1. We assume that $\varphi_{1}$ and $\varphi_{2}$ fulfil the condition (1.2), and the coefficients $a_{i}$, $b_{i}, i=1,2$, and $c$ fulfil the condition (1.3), then the operator

$$
\mathcal{L}=\partial_{t}-\sum_{i=1}^{2} a_{i}\left(t, x_{1}, x_{2}\right) \partial_{i i}+\sum_{i=1}^{2} b_{i}\left(t, x_{1}, x_{2}\right) \partial_{i}+c\left(t, x_{1}, x_{2}\right)
$$

is an isomorphism from $\mathcal{H}_{0}^{1,2}(Q)$ into $L^{2}(Q)$.

The case $a_{1}=a_{2}=1, b_{1}=b_{2}=c=0$, corresponding to the heat operator has been studied in [15] and [17] both in bi-dimensional and multidimensional cases.

Whereas parabolic equations with variables coefficients in cylindrical domains are well studied, the literature concerning such problems in non-cylindrical domains does not seem to be very rich, see [24] for the case of smooth coefficients and [28] for the case of discontinuous coefficients. Concerning parabolic equations in time-varying domains we can find in Fichera [9] and Oleinik [29] solvability results for non-divergence parabolic equations. For the divergence form case, see [5,14] and [25]. In the case of Hölder spaces functional framework, we can find in Baderko [4] results for non-cylindrical domains of the same kind but which cannot include our domain. In [10], we can find Wiener type criterion in the framework of continuous spaces which cannot include our $L^{2}$-case.

Our work is motivated by the interest of researchers for many mathematical questions related to non-regular domains. During the last decades and since many applied problems lead directly to boundary-value problems in "bad" domains, numerous authors studied partial differential equations in non-smooth domains. Among these we can cite $[7,8,11,12,19,21,22,32]$ and the references therein.

The organization of this paper is as follows. In Section 2, we divide the proof of Theorem 1.1 into three steps:

a) We prove well-posedness results for Problem (1.1) when $Q$ is replaced by the truncated domain

$$
\left.Q_{\alpha}=\left\{\left(t, x_{1}\right) \in \mathbb{R}^{2}: \alpha<t<T ; \varphi_{1}(t)<x_{1}<\varphi_{2}(t)\right\} \times\right] 0, b[,
$$

with $\alpha>0$, (Theorem 2.1).

b) We approximate $Q$ by a sequence $\left(Q_{n}\right), n \in \mathbb{N}^{*}$, of such truncated regular domains and we establish a uniform estimate (see Proposition 2.1) of the type

$$
\left\|u_{n}\right\|_{\mathcal{H}^{1,2}\left(Q_{n}\right)} \leqslant K\|f\|_{L^{2}(Q)},
$$

where $u_{n}$ is the solution of Problem (1.1) in $Q_{n}$ and $K$ is a constant independent of $n$.

c) We build a solution $u$ of Problem (1.1), by considering $\widetilde{u_{n}}$ the 0 -extension to $Q$ of the solutions $u_{n}\left(u_{n}, n \in \mathbb{N}^{*}\right.$ exists by Theorem 2.1), and showing (in virtue of Proposition 2.1) that $\widetilde{u_{n_{k}}} \rightarrow u$, weakly in $L^{2}(Q)$, for a suitable increasing sequence of integers $\left(n_{k}\right)_{k \geqslant 1}$. 
Note that this work may be extended at least in the following directions:

1. The function $f$ on the right-hand side of the equation of Problem (1.1), may be taken in $\left.L^{p}(Q), p \in\right] 1, \infty[$. The domain decomposition method used here does not seem to be appropriate for the space $L^{p}(Q)$ when $p \neq 2$. An idea for this extension can be found in [13] or in [23].

2 . The bi-dimensional case in $x$, can be naturally extended to an upper dimension in $x$, such as, for example, the following problem

$$
\partial_{t} u-\sum_{i=1}^{N} a_{i}\left(t, x_{1}, \ldots, x_{N}\right) \partial_{i i} u+\sum_{i=1}^{N} b_{i}\left(t, x_{1}, \ldots, x_{N}\right) \partial_{i} u+c\left(t, x_{1}, \ldots, x_{N}\right) u=f\left(t, x_{1}, \ldots, x_{N}\right),
$$

in the domain

$$
\left\{\left(t, x_{1}, \ldots, x_{N}\right) \in \mathbb{R}^{N+1}: 0<t<T, 0 \leqslant \sqrt{x_{1}^{2}+\ldots+x_{N}^{2}}<\varphi(t)\right\}, N \geqslant 2 .
$$

These questions will be developed in forthcoming works.

\section{Proof of Theorem 1.1}

We divide the proof of Theorem 1.1 into three steps.

\subsection{Step 1: case of a truncated domain $Q_{\alpha}$ which can be transformed into a parallelepiped}

In this subsection, we replace $Q$ by

$$
\left.Q_{\alpha}=\left\{\left(t, x_{1}\right) \in \mathbb{R}^{2}: \alpha<t<T ; \varphi_{1}(t)<x_{1}<\varphi_{2}(t)\right\} \times\right] 0, b[,
$$

with $\alpha>0$, (see, Fig. 2). Thus, we have $\varphi(\alpha)>0$.

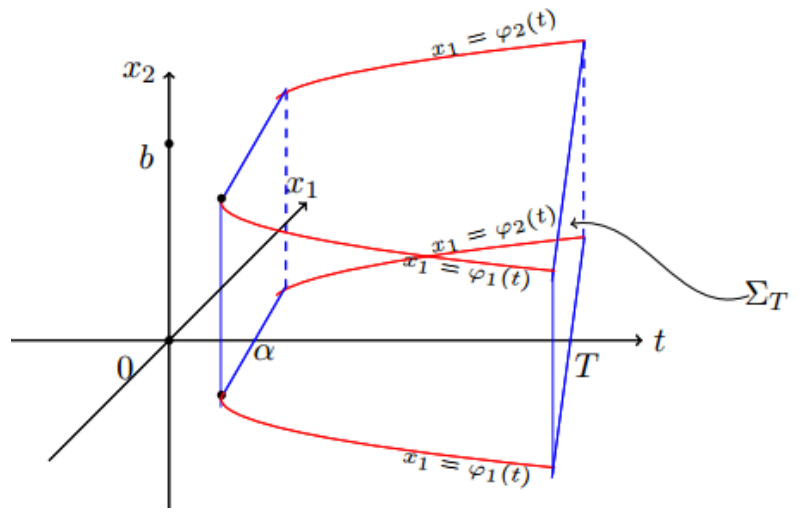

Fig. 2. The truncated domain $Q_{\alpha}$

We can find a change of variable $\psi$ mapping $Q_{\alpha}$ into the parallelepiped

$$
\left.P_{\alpha}=\right] \alpha, T[\times] 0,1[\times] 0, b[
$$

which leaves the variable $t$ unchanged. $\psi$ is defined as follows:

$$
\begin{aligned}
& \psi: Q_{\alpha} \longrightarrow P_{\alpha} \\
&\left(t, x_{1}, x_{2}\right) \longmapsto \psi\left(t, x_{1}, x_{2}\right)=\left(t, y_{1}, y_{2}\right)=\left(t, \frac{x_{1}-\varphi_{1}(t)}{\varphi(t)}, x_{2}\right) . \\
&-419-
\end{aligned}
$$


The mapping $\psi$ transforms the parabolic equation in the domain $Q_{\alpha}$ into a variable-coefficient parabolic equation in the parallelepiped $P_{\alpha}$. Indeed, the equation

$$
\partial_{t} u-\sum_{i=1}^{2} a_{i}\left(t, x_{1}, x_{2}\right) \partial_{i i} u+\sum_{i=1}^{2} b_{i}\left(t, x_{1}, x_{2}\right) \partial_{i} u+c\left(t, x_{1}, x_{2}\right) u=f\left(t, x_{1}, x_{2}\right)
$$

in $Q_{\alpha}$ is equivalent to the following

$$
\partial_{t} v-\sum_{i=1}^{2} a_{i} \widetilde{\left(t, y_{1}, y_{2}\right)} \partial_{i i} v+\sum_{i=1}^{2} b_{i} \widetilde{\left(t, y_{1}, y_{2}\right)} \partial_{i} v+c \widetilde{\left(t, y_{1}, y_{2}\right)} v=g\left(t, y_{1}, y_{2}\right)
$$

in $P_{\alpha}$, where $\left.a_{i} \widetilde{\left(t, y_{1}, y_{2}\right.}\right), b_{i} \widetilde{\left(t, y_{1}, y_{2}\right)}$ and $\left.c \widetilde{\left(t, y_{1}, y_{2}\right.}\right)$ are defined by

$$
\begin{aligned}
& \left.a_{1} \widetilde{\left(t, y_{1}, y_{2}\right)}=\frac{a_{1}\left(t, \varphi(t) y_{1}+\varphi_{1}(t), y_{2}\right)}{\varphi^{2}(t)}, \quad \widetilde{a_{2}} \widetilde{\left(t, y_{1}, y_{2}\right.}\right)=a_{2}\left(t, \varphi(t) y_{1}+\varphi_{1}(t), y_{2}\right), \\
& b_{1} \widetilde{\left(t, y_{1}, y_{2}\right)}=\frac{b_{1}\left(t, \varphi(t) y_{1}+\varphi_{1}(t), y_{2}\right)}{\varphi(t)}\left[1-\varphi^{\prime}(t) y_{1}-\varphi_{1}^{\prime}(t)\right] \text {, } \\
& b_{2} \widetilde{\left(t, y_{1}, y_{2}\right)}=b_{2}\left(t, \varphi(t) y_{1}+\varphi_{1}(t), y_{2}\right), \quad c\left(\widetilde{t, y_{1}, y_{2}}\right)=c\left(t, \varphi(t) y_{1}+\varphi_{1}(t), y_{2}\right),
\end{aligned}
$$

and

$$
g\left(t, y_{1}, y_{2}\right)=f\left(t, x_{1}, x_{2}\right), v\left(t, y_{1}, y_{2}\right)=u\left(t, x_{1}, x_{2}\right) .
$$

Since the functions $a_{i}, b_{i}, i=1,2, c$ and $\varphi$ are bounded, and using the fact that the mapping $\psi$ is tri-Lipschitz, then, it is easy to check the following

Lemma 2.1. $u \in \mathcal{H}^{1,2}\left(Q_{\alpha}\right)$ if and only if $v \in \mathcal{H}^{1,2}\left(P_{\alpha}\right)$.

The boundary conditions on $v$ which correspond to the boundary conditions on $u$ are the following

$$
\left.v\right|_{\partial P_{\alpha} \backslash \Gamma_{T}}=0,
$$

where $\Gamma_{T}$ is the part of the boundary of $P_{\alpha}$ where $t=T$. In the sequel, the variables $\left(t, y_{1}, y_{2}\right)$ will be denoted again by $\left(t, x_{1}, x_{2}\right)$.

Theorem 2.1. The operator

$$
\left.\left.\left.\mathcal{L}^{\prime}=\partial_{t}-\sum_{i=1}^{2} a_{i} \widetilde{\left(t, x_{1},\right.}, x_{2}\right) \partial_{i i}+\sum_{i=1}^{2} b_{i} \widetilde{\left(t, x_{1}, x_{2}\right.}\right) \partial_{i}+c \widetilde{\left(t, x_{1}, x_{2}\right.}\right)
$$

is an isomorphism from $\mathcal{H}_{0}^{1,2}\left(P_{\alpha}\right)$ into $L^{2}\left(P_{\alpha}\right)$, with

$$
\mathcal{H}_{0}^{1,2}\left(P_{\alpha}\right)=\left\{v \in \mathcal{H}^{1,2}\left(P_{\alpha}\right):\left.v\right|_{\partial P_{\alpha} \backslash \Gamma_{T}}=0\right\} .
$$

Proof. Since the differentiable coefficients $a_{i}\left(\widetilde{t, x_{1}}, x_{2}\right), b_{i}\left(\widetilde{t, x_{1}}, x_{2}\right), i=1,2$ and $c\left(\widetilde{\left.t, x_{1}, x_{2}\right)}\right.$ are bounded in $\overline{P_{\alpha}}$, the optimal regularity is given by Ladyzhenskaya-Solonnikov-Ural'tseva [24].

We shall need the following result in order to justify all the calculus of the next subsection.

Lemma 2.2. The space

is dense in the space

$$
\left\{v \in H^{4}\left(P_{\alpha}\right):\left.v\right|_{\partial_{p} P_{\alpha}}=0\right\}
$$

$$
\left\{v \in \mathcal{H}^{1,2}\left(P_{\alpha}\right):\left.v\right|_{\partial_{p} P_{\alpha}}=0\right\}
$$

Here, $\partial_{p} P_{\alpha}$ is the parabolic boundary of $P_{\alpha}$ and $H^{4}$ stands for the usual Sobolev space defined, for instance, in Lions-Magenes [26]. 
The proof of the above lemma may be found in [15].

Remark 2.1. In Lemma 2.2, we can replace $P_{\alpha}$ by $Q_{\alpha}$ with the help of the change of variable $\psi$ defined above.

\subsection{Step 2: uniform estimate}

We denote $u_{n} \in \mathcal{H}^{1,2}\left(Q_{n}\right), n \in \mathbb{N}^{*}$, the solution of Problem (1.1) corresponding to a second member $f_{n}=\left.f\right|_{Q_{n}} \in L^{2}\left(Q_{n}\right)$ in

$$
\left.Q_{n}=\left\{\left(t, x_{1}\right) \in \mathbb{R}^{2}: \frac{1}{n}<t<T, \varphi_{1}(t)<x_{1}<\varphi_{2}(t)\right\} \times\right] 0, b[.
$$

Proposition 2.1. There exists a constant $K_{1}$ independent of $n$ such that

$$
\left\|u_{n}\right\|_{\mathcal{H}^{1,2}\left(Q_{n}\right)} \leqslant K_{1}\left\|f_{n}\right\|_{L^{2}\left(Q_{n}\right)} \leqslant K_{1}\|f\|_{L^{2}(Q)} .
$$

In order to prove Proposition 2.1, we need some preliminary results.

Lemma 2.3. Let $] \alpha, \beta\left[\subset \mathbb{R}\right.$. There exists a constant $K_{2}$ (independent of $\alpha$ and $\beta$ ) such that

$$
\left\|w^{(j)}\right\|_{L^{2}(] \alpha, \beta[)}^{2} \leqslant K_{2}(\beta-\alpha)^{2(2-j)}\left\|w^{(2)}\right\|_{L^{2}(] \alpha, \beta[)}^{2}, j=0,1,
$$

for every $w \in H^{2}(] \alpha, \beta[) \cap H_{0}^{1}(] \alpha, \beta[)$, where $w^{(j)}, j=1,2$, denotes the derivative of order $j$ of $w$ on $] \alpha, \beta\left[\right.$ and $w^{(0)}=w$.

Lemma 2.4. For every $\epsilon>0$, chosen such that $\varphi(t) \leqslant \epsilon$, there exists a constant $C_{1}$ independent of $n$ such that for $i=1,2$

$$
\left\|\partial_{i}^{j} u_{n}\right\|_{L^{2}\left(Q_{n}\right)}^{2} \leqslant C_{1} \epsilon^{2(2-j)}\left\|\partial_{i i} u_{n}\right\|_{L^{2}\left(Q_{n}\right)}^{2}, \quad j=0,1
$$

where $\partial_{i}^{1} u_{n}=\partial_{i} u_{n}$ and $\partial_{i}^{0} u_{n}=u_{n}$.

Proof. Replacing in Lemma $2.3 w$ by $u_{n}$ and $] \alpha, \beta[$ by $] \varphi_{1}(t), \varphi_{2}(t)[$, for a fixed $t$, we obtain

$$
\begin{aligned}
\int_{\varphi_{1}(t)}^{\varphi_{2}(t)}\left(\partial_{i}^{j} u_{n}\right)^{2} d x_{1} & \leqslant K_{2}(\varphi(t))^{2(2-j)} \int_{\varphi_{1}(t)}^{\varphi_{2}(t)}\left(\partial_{i i} u_{n}\right)^{2} d x_{1} \leqslant \\
& \leqslant K_{2} \epsilon^{2(2-j)} \int_{\varphi_{1}(t)}^{\varphi_{2}(t)}\left(\partial_{i i} u_{n}\right)^{2} d x_{1},
\end{aligned}
$$

with $i=1,2$ and $j=0,1$. Integrating in the previous inequality with respect to $t$, then with respect to $x_{2}$, we get the desired result with $C_{1}=K_{2}$.

Proof of Proposition 2.1. Let us denote the inner product in $L^{2}\left(Q_{n}\right)$ by $\langle.,$.$\rangle , then we have$

$$
\begin{aligned}
& \left\|f_{n}\right\|_{L^{2}\left(Q_{n}\right)}^{2}=\left\|\partial_{t} u_{n}-\sum_{i=1}^{2} a_{i}\left(t, x_{1}, x_{2}\right) \partial_{i i} u_{n}+\sum_{i=1}^{2} b_{i}\left(t, x_{1}, x_{2}\right) \partial_{i} u_{n}+c\left(t, x_{1}, x_{2}\right) u_{n}\right\|_{L^{2}\left(Q_{n}\right)}^{2}= \\
& =\left\|\partial_{t} u_{n}\right\|_{L^{2}\left(Q_{n}\right)}^{2}+\sum_{i=1}^{2}\left\|a_{i} \partial_{i i} u_{n}\right\|_{L^{2}\left(Q_{n}\right)}^{2}+\sum_{i=1}^{2}\left\|b_{i} \partial_{i} u_{n}\right\|_{L^{2}\left(Q_{n}\right)}^{2}+\left\|c u_{n}\right\|_{L^{2}\left(Q_{n}\right)}^{2}- \\
& -2 \sum_{i=1}^{2}\left\langle\partial_{t} u_{n}, a_{i} \partial_{i i} u_{n}\right\rangle+2 \sum_{i=1}^{2}\left\langle\partial_{t} u_{n}, b_{i} \partial_{i} u_{n}\right\rangle+2\left\langle\partial_{t} u_{n}, c u_{n}\right\rangle-2 \sum_{i=1}^{2}\left\langle a_{i} \partial_{i i} u_{n}, b_{1} \partial_{1} u_{n}\right\rangle- \\
& -2 \sum_{i=1}^{2}\left\langle a_{i} \partial_{i i} u_{n}, b_{2} \partial_{2} u_{n}\right\rangle-2 \sum_{i=1}^{2}\left\langle a_{i} \partial_{i i} u_{n}, c u_{n}\right\rangle+2 \sum_{i=1}^{2}\left\langle b_{i} \partial_{i} u_{n}, c u_{n}\right\rangle+ \\
& +2\left\langle a_{11} \partial_{11} u_{n}, a_{22} \partial_{22} u_{n}\right\rangle-2\left\langle b_{1} \partial_{1} u_{n}, b_{2} \partial_{2} u_{n}\right\rangle .
\end{aligned}
$$


1) Estimation of $-2\left\langle\partial_{t} u_{n}, a_{i} \partial_{i i} u_{n}\right\rangle, i=1,2:$ We have

$$
\partial_{t} u_{n} \partial_{i i} u_{n}=\partial_{i}\left(\partial_{t} u_{n} \partial_{i} u_{n}\right)-\frac{1}{2} \partial_{t}\left(\partial_{i} u_{n}\right)^{2}
$$

Then

$$
\begin{aligned}
-2\left\langle\partial_{t} u_{n}, a_{i} \partial_{i i} u_{n}\right\rangle= & -2 \int_{Q_{n}} a_{i} \partial_{t} u_{n} \partial_{i i} u_{n} d t d x_{1} d x_{2}= \\
= & \int_{Q_{n}} a_{i}\left[-2 \partial_{i}\left(\partial_{t} u_{n} \partial_{i} u_{n}\right)+\partial_{t}\left(\partial_{i} u_{n}\right)^{2}\right] d t d x_{1} d x_{2}= \\
= & \int_{\partial Q_{n}} a_{i}\left[\left(\partial_{i} u_{n}\right)^{2} \nu_{t}-2 \partial_{t} u_{n} \partial_{i} u_{n} \nu_{i}\right] d \sigma+ \\
& +\int_{Q_{n}}\left[2 \partial_{i} a_{i i}\left(\partial_{t} u_{n} \partial_{i} u_{n}\right)-\partial_{t} a_{i i}\left(\partial_{i} u_{n}\right)^{2}\right] d t d x_{1} d x_{2},
\end{aligned}
$$

where $\nu_{t}, \nu_{i}, i=1,2$ are the components of the unit outward normal vector at $\partial Q_{n}$. We shall rewrite the boundary integral making use of the boundary conditions. On the parts of the boundary of $Q_{n}$ where $t=\frac{1}{n}, x_{2}=0$ and $x_{2}=b$ we have $u_{n}=0$ and consequently $\partial_{i} u_{n}=0$. The corresponding boundary integral vanishes. On the part of the boundary where $t=T$, we have $\nu_{i}=0$ and $\nu_{t}=1$. Accordingly the corresponding boundary integral

$$
\int_{0}^{b} \int_{\varphi_{1}(T)}^{\varphi_{2}(T)} a_{i}\left(T, x_{1}, x_{2}\right)\left(\partial_{i} u_{n}\right)^{2} d x_{1} d x_{2}
$$

is nonnegative, since $a_{i}\left(T, x_{1}, x_{2}\right)>0$. On the part of the boundary where $x_{1}=\varphi_{k}(t), k=1,2$, we have

$$
\nu_{1}=\frac{(-1)^{k}}{\sqrt{1+\left(\varphi_{k}^{\prime}\right)^{2}(t)}}, \quad \nu_{t}=\frac{(-1)^{k+1} \varphi_{k}^{\prime}(t)}{\sqrt{1+\left(\varphi_{k}^{\prime}\right)^{2}(t)}} \text { and } \nu_{2}=0
$$

Consequently, the corresponding boundary integral is

$$
I_{n, i}=\sum_{k=1}^{2}(-1)^{k+i+1} \int_{0}^{b} \int_{\frac{1}{n}}^{T} a_{i}\left(t, \varphi_{k}(t), x_{2}\right) \varphi_{k}^{\prime}(t)\left[\partial_{i} u_{n}\left(t, \varphi_{k}(t), x_{2}\right)\right]^{2} d t d x_{2} .
$$

Furthermore,

$$
\left|\int_{Q_{n}} \partial_{t} a_{i}\left(\partial_{i} u_{n}\right)^{2} d t d x_{1} d x_{2}\right| \leqslant c_{1}\left\|\partial_{i} u_{n}\right\|_{L^{2}\left(Q_{n}\right)}^{2},
$$

and for every $\epsilon>0$

$$
\begin{aligned}
\left|\int_{Q_{n}} \partial_{i} a_{i}\left(\partial_{t} u_{n} \partial_{i} u_{n}\right) d t d x_{1} d x_{2}\right| & \leqslant c_{1} \int_{Q_{n}}\left|\partial_{t} u_{n}\right|\left|\partial_{i} u_{n}\right| d t d x_{1} d x_{2} \leqslant \\
& \leqslant c_{1} \frac{\epsilon}{2}\left\|\partial_{t} u_{n}\right\|_{L^{2}\left(Q_{n}\right)}^{2}+\frac{c_{1}}{2 \epsilon}\left\|\partial_{i} u_{n}\right\|_{L^{2}\left(Q_{n}\right)}^{2} .
\end{aligned}
$$

Then for $i=1,2$ we have

$$
-2\left\langle\partial_{t} u_{n}, \partial_{i} u_{n}\right\rangle \geqslant-\left|I_{n, 1, i}\right|-\left|I_{n, 2, i}\right|-c_{1}\left\|\partial_{i} u_{n}\right\|_{L^{2}\left(Q_{n}\right)}^{2}-c_{1} \epsilon\left\|\partial_{t} u_{n}\right\|_{L^{2}\left(Q_{n}\right)}^{2}-\frac{c_{1}}{\epsilon}\left\|\partial_{i} u_{n}\right\|_{L^{2}\left(Q_{n}\right)}^{2}
$$

where

$$
I_{n, k, i}=(-1)^{k+i+1} \int_{0}^{b} \int_{\frac{1}{n}}^{T} a_{i}\left(t, \varphi_{k}(t), x_{2}\right) \varphi_{k}^{\prime}(t)\left[\partial_{i} u_{n}\left(t, \varphi_{k}(t), x_{2}\right)\right]^{2} d t d x_{2}, k=1,2 .
$$


Lemma 2.5. There exists a positive constant $K_{4}$ independent of $n$ such that

$$
\begin{aligned}
& \left|I_{n, k, 1}\right| \leqslant K_{4} \epsilon\left\|\partial_{11} u_{n}\right\|_{L^{2}\left(Q_{n}\right)}^{2}, k=1,2, \\
& \left|I_{n, k, 2}\right| \leqslant K_{4} \epsilon\left\|\partial_{22} u_{n}\right\|_{L^{2}\left(Q_{n}\right)}^{2}+c_{0} \epsilon\left\|\partial_{12} u_{n}\right\|_{L^{2}\left(Q_{n}\right)}^{2}, k=1,2,
\end{aligned}
$$

where $\partial_{12} u_{n}=\frac{\partial^{2} u_{n}}{\partial x_{1} \partial x_{2}}$.

Proof. We convert the boundary integral $I_{n, 1,1}$ into a surface integral by setting

$$
\begin{aligned}
{\left[\partial_{1} u_{n}\left(t, \varphi_{1}(t), x_{2}\right)\right]^{2} } & =-\left.\frac{\varphi_{2}(t)-x_{1}}{\varphi(t)}\left[\partial_{1} u_{n}\left(t, x_{1}, x_{2}\right)\right]^{2}\right|_{x_{1}=\varphi_{1}(t)} ^{x_{1}=\varphi_{2}(t)}= \\
& =-\int_{\varphi_{1}(t)}^{\varphi_{2}(t)} \partial_{1}\left\{\frac{\varphi_{2}(t)-x_{1}}{\varphi(t)}\left[\partial_{1} u_{n}\right]^{2}\right\} d x_{1}= \\
& =-2 \int_{\varphi_{1}(t)}^{\varphi_{2}(t)} \frac{\varphi_{2}(t)-x_{1}}{\varphi(t)} \partial_{1} u_{n} . \partial_{11} u_{n} d x_{1}+\int_{\varphi_{1}(t)}^{\varphi_{2}(t)} \frac{1}{\varphi(t)}\left[\partial_{1} u_{n}\right]^{2} d x_{1} .
\end{aligned}
$$

Then, we have

$$
\begin{aligned}
I_{n, 1,1}= & -\int_{0}^{b} \int_{\frac{1}{n}}^{T} a_{1}\left(t, \varphi_{1}(t), x_{2}\right) \varphi_{1}^{\prime}(t)\left[\partial_{1} u_{n}\left(t, \varphi_{1}(t), x_{2}\right)\right]^{2} d t d x_{2}= \\
= & -\int_{Q_{n}} a_{1}\left(t, \varphi_{1}(t), x_{2}\right) \frac{\varphi_{1}^{\prime}(t)}{\varphi(t)}\left(\partial_{1} u_{n}\right)^{2} d t d x_{1} d x_{2}+ \\
& +2 \int_{Q_{n}} a_{1}\left(t, \varphi_{1}(t), x_{2}\right) \frac{\varphi_{2}(t)-x_{1}}{\varphi(t)} \varphi_{1}^{\prime}(t)\left(\partial_{1} u_{n}\right)\left(\partial_{11} u_{n}\right) d t d x_{1} d x_{2}
\end{aligned}
$$

Thanks to Lemma 2.4, we can write

$$
\int_{\varphi_{1}(t)}^{\varphi_{2}(t)}\left[\partial_{1} u_{n}\right]^{2} d x_{1} \leqslant K_{2} \varphi(t)^{2} \int_{\varphi_{1}(t)}^{\varphi_{2}(t)}\left[\partial_{11} u_{n}\right]^{2} d x_{1}
$$

Therefore

$$
\int_{\varphi_{1}(t)}^{\varphi_{2}(t)}\left[\partial_{1} u_{n}\right]^{2} \frac{\left|\varphi_{1}^{\prime}\right|}{\varphi} d x_{1} \leqslant K_{2}\left|\varphi_{1}^{\prime}\right| \varphi \int_{\varphi_{1}(t)}^{\varphi_{2}(t)}\left[\partial_{11} u_{n}\right]^{2} d x_{1}
$$

consequently

$$
\left|I_{n, 1,1}\right| \leqslant K_{2} \int_{Q_{n}} c_{0}\left|\varphi_{1}^{\prime}\right| \varphi\left(\partial_{11} u_{n}\right)^{2} d t d x_{1} d x_{2}+2 \int_{Q_{n}} c_{0}\left|\varphi_{1}^{\prime}\right|\left|\partial_{1} u_{n}\right|\left|\partial_{11} u_{n}\right| d t d x_{1} d x_{2}
$$

since $\left|\frac{\varphi_{2}(t)-x_{1}}{\varphi(t)}\right| \leqslant 1$. Using the inequality

$$
2\left|\varphi_{1}^{\prime} \partial_{1} u_{n}\right|\left|\partial_{11} u_{n}\right| \leqslant \epsilon\left(\partial_{11} u_{n}\right)^{2}+\frac{1}{\epsilon}\left(\varphi_{1}^{\prime}\right)^{2}\left(\partial_{1} u_{n}\right)^{2}
$$

for all $\epsilon>0$, we obtain

$$
\left|I_{n, 1,1}\right| \leqslant K_{2} \int_{Q_{n}}\left[c_{0}\left|\varphi_{1}^{\prime}\right| \varphi+c_{0} \epsilon\right]\left(\partial_{11} u_{n}\right)^{2} d t d x_{1} d x_{2}+\frac{c_{0}}{\epsilon} \int_{Q_{n}}\left(\varphi_{1}^{\prime}\right)^{2}\left(\partial_{1} u_{n}\right)^{2} d t d x_{1} d x_{2}
$$


Lemma 2.4 yields

$$
\frac{1}{\epsilon} \int_{Q_{n}}\left(\varphi_{1}^{\prime}\right)^{2}\left(\partial_{1} u_{n}\right)^{2} d t d x_{1} d x_{2} \leqslant K_{2} \frac{1}{\epsilon} \int_{Q_{n}}\left(\varphi_{1}^{\prime}\right)^{2} \varphi^{2}\left(\partial_{11} u_{n}\right)^{2} d t d x_{1} d x_{2}
$$

Thus,

$$
\begin{aligned}
\left|I_{n, 1,1}\right| & \leqslant K_{2} \int_{Q_{n}} c_{0}\left[\left|\varphi_{1}^{\prime}\right||\varphi|+\frac{1}{\epsilon}\left(\varphi_{1}^{\prime}\right)^{2}|\varphi|^{2}\right]\left(\partial_{11} u_{n}\right)^{2} d t d x_{1} d x_{2}+\int_{Q_{n}} c_{0} \epsilon\left(\partial_{11} u_{n}\right)^{2} d t d x_{1} d x_{2} \leqslant \\
& \leqslant\left(2 K_{2}+1\right) c_{0} \epsilon \int_{Q_{n}}\left(\partial_{11} u_{n}\right)^{2} d t d x_{1} d x_{2},
\end{aligned}
$$

since $\left|\varphi_{1}^{\prime} \varphi\right| \leqslant \epsilon$. Finally, taking $K_{4}=\left(2 K_{2}+1\right) c_{0}$, we obtain

$$
\left|I_{n, 1,1}\right| \leqslant K_{4} \epsilon\left\|\partial_{11} u_{n}\right\|_{L^{2}\left(Q_{n}\right)}^{2} .
$$

The inequalities

$$
\left|I_{n, 2,1}\right| \leqslant K_{4} \epsilon\left\|\partial_{11} u_{n}\right\|_{L^{2}\left(Q_{n}\right)}^{2}
$$

and

$$
\left|I_{n, k, 2}\right| \leqslant K_{4} \epsilon\left\|\partial_{22} u_{n}\right\|_{L^{2}\left(Q_{n}\right)}^{2}+c_{0} \epsilon\left\|\partial_{12} u_{n}\right\|_{L^{2}\left(Q_{n}\right)}^{2}, \quad k=1,2
$$

can be proved by a similar method. This ends the proof of Lemma 2.5 .

2) Estimation of $2\left\langle a_{1} \partial_{11} u_{n}, a_{2} \partial_{22} u_{n}\right\rangle$ : We have

$$
\partial_{11} u_{n} . \partial_{22} u_{n}=\partial_{1}\left(\partial_{1} u_{n} . \partial_{22} u_{n}\right)-\partial_{2}\left(\partial_{1} u_{n} . \partial_{12} u_{n}\right)+\left(\partial_{12} u_{n}\right)^{2} .
$$

Then

$$
\begin{aligned}
2\left\langle a_{1} \partial_{11} u_{n}, a_{2} \partial_{22} u_{n}\right\rangle & =2 \int_{Q_{n}} a_{1} a_{2} \partial_{11} u_{n} . \partial_{22} u_{n} d t d x_{1} d x_{2}= \\
& =2 \int_{Q_{n}} a_{1} a_{2}\left[\partial_{1}\left(\partial_{1} u_{n} . \partial_{22} u_{n}\right)-\partial_{2}\left(\partial_{1} u_{n} . \partial_{12} u_{n}\right)+\left(\partial_{12} u_{n}\right)^{2}\right] d t d x_{1} d x_{2}= \\
& =2 \int_{\partial Q_{n}} a_{1} a_{2}\left[\partial_{1} u_{n} . \partial_{22} u_{n} \nu_{1}-\partial_{1} u_{n} . \partial_{12} u_{n} \nu_{2}\right] d \sigma+ \\
& +2 \int_{Q_{n}} a_{1} a_{2}\left(\partial_{12} u_{n}\right)^{2} d t d x_{1} d x_{2}- \\
& -2 \int_{Q_{n}} \partial_{1}\left(a_{1} a_{2}\right) \cdot\left(\partial_{1} u_{n} . \partial_{22} u_{n}\right) d t d x_{1} d x_{2}+ \\
& +2 \int_{Q_{n}} \partial_{2}\left(a_{1} a_{2}\right)\left(\partial_{1} u_{n} . \partial_{12} u_{n}\right) d t d x_{1} d x_{2}
\end{aligned}
$$

where $\nu_{t}, \nu_{i}, i=1,2$ are the components of the unit outward normal vector at $\partial Q_{n}$. We shall rewrite the boundary integral making use of the boundary conditions. On the parts of the boundary of $Q_{n}$ where $t=\frac{1}{n}, x_{2}=0$ and $x_{2}=b$ we have $u_{n}=0$ and consequently $\partial_{1} u_{n}=0$. The corresponding boundary integral vanishes. On the part of the boundary where $t=T$, we have $\nu_{1}=\nu_{2}=0$. Accordingly the corresponding boundary integral vanishes. On the part of the boundary where $x_{1}=\varphi_{k}(t), k=1,2$, we have $\nu_{2}=0, u_{n}=0$ and consequently $\partial_{22} u_{n}=0$. The corresponding boundary integral vanishes. So,

$$
2 \int_{\partial Q_{n}} a_{1} a_{2}\left[\partial_{1} u_{n} . \partial_{22} u_{n} \nu_{1}-\partial_{1} u_{n} . \partial_{12} u_{n} \nu_{2}\right] d \sigma=0
$$


Furthermore,

$$
2 \int_{Q_{n}} a_{1} a_{2}\left(\partial_{12} u_{n}\right)^{2} d t d x_{1} d x_{2} \geqslant 2 a_{0}\left\|\partial_{12} u_{n}\right\|_{L^{2}\left(Q_{n}\right)}^{2}
$$

and for every $\epsilon>0$

$$
\begin{aligned}
& -2 \int_{Q_{n}} \partial_{1}\left(a_{1} a_{2}\right) \cdot\left(\partial_{1} u_{n} \cdot \partial_{22} u_{n}\right) d t d x_{1} d x_{2} \geqslant-\beta_{1} \epsilon\left\|\partial_{22} u_{n}\right\|_{L^{2}\left(Q_{n}\right)}^{2}-\frac{\beta_{1}}{\epsilon}\left\|\partial_{1} u_{n}\right\|_{L^{2}\left(Q_{n}\right)}^{2}, \\
& +2 \int_{Q_{n}} \partial_{2}\left(a_{1} a_{2}\right)\left(\partial_{1} u_{n} \cdot \partial_{12} u_{n}\right) d t d x_{1} d x_{2} \geqslant-\beta_{1} \epsilon\left\|\partial_{12} u_{n}\right\|_{L^{2}\left(Q_{n}\right)}^{2}-\frac{\beta_{1}}{\epsilon}\left\|\partial_{1} u_{n}\right\|_{L^{2}\left(Q_{n}\right)}^{2}
\end{aligned}
$$

with $\beta_{1}$ is a positive constant. Then, we have

$$
2\left\langle a_{1} \partial_{11} u_{n}, a_{2} \partial_{22} u_{n}\right\rangle \geqslant\left(2 a_{0}-\beta_{1} \epsilon\right)\left\|\partial_{12} u_{n}\right\|_{L^{2}\left(Q_{n}\right)}^{2}-\beta_{1} \epsilon\left\|\partial_{22} u_{n}\right\|_{L^{2}\left(Q_{n}\right)}^{2}-\frac{2 \beta_{1}}{\epsilon}\left\|\partial_{1} u_{n}\right\|_{L^{2}\left(Q_{n}\right)}^{2} .
$$

It is easy to establish the following estimates.

Lemma 2.6. Set $c_{4}=c_{0} c_{2}, c_{5}=c_{0} c_{3}$ and $c_{6}=c_{2} c_{3}$. Then, for every $\epsilon>0$ we have

$$
\begin{aligned}
& 2\left\langle\partial_{t} u_{n}, b_{i} \partial_{i} u_{n}\right\rangle \geqslant-\epsilon c_{2}\left\|\partial_{t} u_{n}\right\|_{L^{2}\left(Q_{n}\right)}^{2}-\frac{c_{2}}{\epsilon}\left\|\partial_{i} u_{n}\right\|_{L^{2}\left(Q_{n}\right)}^{2}, i=1,2, \\
& 2\left\langle\partial_{t} u_{n}, c u_{n}\right\rangle \geqslant-\epsilon c_{3}\left\|\partial_{t} u_{n}\right\|_{L^{2}\left(Q_{n}\right)}^{2}-\frac{c_{3}}{\epsilon}\left\|u_{n}\right\|_{L^{2}\left(Q_{n}\right)}^{2}, \\
&-2\left\langle a_{i} \partial_{i i} u_{n}, b_{k} \partial_{k} u_{n}\right\rangle \geqslant-c_{4} \epsilon\left\|\partial_{i i} u_{n}\right\|_{L^{2}\left(Q_{n}\right)}^{2}-\frac{c_{4}}{\epsilon}\left\|\partial_{k} u_{n}\right\|_{L^{2}\left(Q_{n}\right)}^{2}, i=1,2 ; k=1,2, \\
&-2\left\langle a_{i} \partial_{i i} u_{n}, c u_{n}\right\rangle \geqslant-c_{5} \epsilon\left\|\partial_{i i} u_{n}\right\|_{L^{2}\left(Q_{n}\right)}^{2}-\frac{c_{5}}{\epsilon}\left\|u_{n}\right\|_{L^{2}\left(Q_{n}\right)}^{2}, i=1,2, \\
& 2\left\langle b_{i} \partial_{i} u_{n}, c u_{n}\right\rangle \geqslant-c_{6} \epsilon\left\|u_{n}\right\|_{L^{2}\left(Q_{n}\right)}^{2}-\frac{c_{6}}{\epsilon}\left\|\partial_{i} u_{n}\right\|_{L^{2}\left(Q_{n}\right)}^{2}, \\
& 2\left\langle b_{1} \partial_{1} u_{n}, b_{2} \partial_{2} u_{n}\right\rangle \geqslant-b_{0} \epsilon\left\|\partial_{1} u_{n}\right\|_{L^{2}\left(Q_{n}\right)}^{2}-\frac{b_{0}}{\epsilon}\left\|\partial_{2} u_{n}\right\|_{L^{2}\left(Q_{n}\right)}^{2} .
\end{aligned}
$$

Now, summing up the estimates (2.1), (2.2) and making use of Lemma 2.5 and Lemma 2.6 then we obtain

$$
\begin{aligned}
\left\|f_{n}\right\|_{L^{2}\left(Q_{n}\right)}^{2} \geqslant & (1-\alpha \epsilon)\left\|\partial_{t} u_{n}\right\|_{L^{2}\left(Q_{n}\right)}^{2}+d_{0}\left\|u_{n}\right\|_{L^{2}\left(Q_{n}\right)}^{2}-\alpha\left(\epsilon+\frac{1}{\epsilon}\right)\left\|u_{n}\right\|_{L^{2}\left(Q_{n}\right)}^{2}+ \\
& +b_{0}\left(\left\|\partial_{1} u_{n}\right\|_{L^{2}\left(Q_{n}\right)}^{2}+\left\|\partial_{2} u_{n}\right\|_{L^{2}\left(Q_{n}\right)}^{2}\right)-\alpha\left(\epsilon+\frac{1}{\epsilon}\right)\left(\left\|\partial_{1} u_{n}\right\|_{L^{2}\left(Q_{n}\right)}^{2}+\left\|\partial_{2} u_{n}\right\|_{L^{2}\left(Q_{n}\right)}^{2}\right)+ \\
& +\left(a_{0}-\alpha \epsilon\right) \sum_{i=1}^{2}\left\|\partial_{i i} u_{n}\right\|_{L^{2}\left(Q_{n}\right)}^{2}+\left(2 a_{0}-\beta_{1} \epsilon-c_{0} \epsilon\right)\left\|\partial_{12} u_{n}\right\|_{L^{2}\left(Q_{n}\right)}^{2}
\end{aligned}
$$

where $\alpha$ is a positive constant independent of $n$. Thanks to Lemma 2.4, it follows that for $i=1,2$

$$
-\alpha\left(\epsilon+\frac{1}{\epsilon}\right)\left\|\partial_{i} u_{n}\right\|_{L^{2}\left(Q_{n}\right)}^{2} \geqslant-\alpha\left(\epsilon+\frac{1}{\epsilon}\right) C_{1} \epsilon^{2}\left\|\partial_{i i} u_{n}\right\|_{L^{2}\left(Q_{n}\right)}^{2}
$$

and

$$
-\alpha\left(\epsilon+\frac{1}{\epsilon}\right)\left\|u_{n}\right\|_{L^{2}\left(Q_{n}\right)}^{2} \geqslant-\alpha\left(\epsilon+\frac{1}{\epsilon}\right) C_{1} \epsilon^{4}\left\|\partial_{i i} u_{n}\right\|_{L^{2}\left(Q_{n}\right)}^{2} .
$$


Therefore,

$$
\begin{aligned}
& \left\|f_{n}\right\|_{L^{2}\left(Q_{n}\right)}^{2} \geqslant(1-\alpha \epsilon)\left\|\partial_{t} u_{n}\right\|_{L^{2}\left(Q_{n}\right)}^{2}+d_{0}\left\|u_{n}\right\|_{L^{2}\left(Q_{n}\right)}^{2}- \\
& -\alpha\left(\epsilon+\frac{2}{\epsilon}\right) C_{1} \epsilon^{4}\left(\left\|\partial_{11} u_{n}\right\|_{L^{2}\left(Q_{n}\right)}^{2}+\left\|\partial_{22} u_{n}\right\|_{L^{2}\left(Q_{n}\right)}^{2}\right)+b_{0}\left(\left\|\partial_{1} u_{n}\right\|_{L^{2}\left(Q_{n}\right)}^{2}+\left\|\partial_{2} u_{n}\right\|_{L^{2}\left(Q_{n}\right)}^{2}\right)- \\
& -\alpha\left(\epsilon+\frac{1}{\epsilon}\right) C_{1} \epsilon^{2}\left(\left\|\partial_{1}^{2} u_{n}\right\|_{L^{2}\left(Q_{n}\right)}^{2}+\left\|\partial_{2} u_{n}\right\|_{L^{2}\left(Q_{n}\right)}^{2}\right)+ \\
& +\left(a_{0}-\alpha \epsilon\right) \sum_{i=1}^{2}\left\|\partial_{i i} u_{n}\right\|_{L^{2}\left(Q_{n}\right)}^{2}+\left(2 a_{0}-\beta_{1} \epsilon-c_{0} \epsilon\right)\left\|\partial_{12} u_{n}\right\|_{L^{2}\left(Q_{n}\right)}^{2},
\end{aligned}
$$

which implies

$$
\begin{aligned}
\left\|f_{n}\right\|_{L^{2}\left(Q_{n}\right)}^{2} \geqslant & (1-\alpha \epsilon)\left\|\partial_{t} u_{n}\right\|_{L^{2}\left(Q_{n}\right)}^{2}+d_{0}\left\|u_{n}\right\|_{L^{2}\left(Q_{n}\right)}^{2}+b_{0}\left(\left\|\partial_{1} u_{n}\right\|_{L^{2}\left(Q_{n}\right)}^{2}+\left\|\partial_{2} u_{n}\right\|_{L^{2}\left(Q_{n}\right)}^{2}\right)+ \\
& +\left(a_{0}-\alpha \epsilon-\alpha C_{1}\left(\epsilon^{2}+\epsilon\right)-\alpha C_{1}\left(\epsilon^{5}+\epsilon^{3}\right)\right)\left(\left\|\partial_{11} u_{n}\right\|_{L^{2}\left(Q_{n}\right)}^{2}+\left\|\partial_{22} u_{n}\right\|_{L^{2}\left(Q_{n}\right)}^{2}\right)+ \\
& +\left(2 a_{0}-\beta_{1} \epsilon-c_{0} \epsilon\right)\left\|\partial_{12} u_{n}\right\|_{L^{2}\left(Q_{n}\right)}^{2}
\end{aligned}
$$

Then, it is sufficient to choose $\epsilon$ verifying

$$
(1-\alpha \epsilon)>0, \quad\left(2 a_{0}-\beta_{1} \epsilon-c_{0} \epsilon\right)>0 \text { and }\left(a_{0}-\alpha \epsilon-\alpha C_{1}\left(\epsilon^{2}+\epsilon\right)-\alpha C_{1}\left(\epsilon^{5}+\epsilon^{3}\right)\right)>0
$$

to get a constant $K_{0}>0$ independent of $n$ such that

$$
\left\|f_{n}\right\|_{L^{2}\left(Q_{n}\right)} \geqslant K_{0}\left\|u_{n}\right\|_{\mathcal{H}^{1,2}\left(Q_{n}\right)},
$$

and since

$$
\left\|f_{n}\right\|_{L^{2}\left(Q_{n}\right)} \leqslant\|f\|_{L^{2}(Q)}
$$

there exists a constant $K_{1}>0$, independent of $n$ satisfying

$$
\left\|u_{n}\right\|_{\mathcal{H}^{1,2}\left(Q_{n}\right)} \leqslant K_{1}\left\|f_{n}\right\|_{L^{2}\left(Q_{n}\right)} \leqslant K_{1}\|f\|_{L^{2}(Q)} .
$$

This completes the proof of Proposition 2.1.

\subsection{Step 3: passage to the limit}

Choose a sequence $Q_{n} n=1,2, \ldots$ of reference domains (see the above subsection) such that $Q_{n} \subseteq Q$. Then we have $Q_{n} \rightarrow Q$, as $n \rightarrow \infty$. Consider the solution $u_{n} \in \mathcal{H}^{1,2}\left(Q_{n}\right)$ of the Cauchy-Dirichlet problem

$$
\left\{\begin{array}{l}
\partial_{t} u_{n}-\sum_{i=1}^{2} a_{i}\left(t, x_{1}, x_{2}\right) \partial_{i i} u_{n}+\sum_{i=1}^{2} b_{i}\left(t, x_{1}, x_{2}\right) \partial_{i} u_{n}+c\left(t, x_{1}, x_{2}\right) u_{n}=f \quad \text { in } Q_{n} \\
\left.u_{n}\right|_{\partial Q_{n} \backslash \Sigma_{T}}=0 .
\end{array}\right.
$$

Such a solution $u_{n}$ exists by Theorem 2.1. Let $\widetilde{u_{n}}$ the 0 -extension of $u_{n}$ to $Q$. In virtue of Proposition 2.1, we know that there exists a constant $C$ such that

$$
\left\|\widetilde{u_{n}}\right\|_{\mathcal{H}^{1,2}\left(Q_{n}\right)} C\|f\|_{L^{2}(Q)} .
$$

This means that $\widetilde{u_{n}}, \widetilde{\partial_{t} u_{n}}, \widetilde{\partial^{\alpha} u_{n}}$ for $1 \leqslant|\alpha| \leqslant 2$ are bounded functions in $L^{2}(Q)$. So, for a suitable increasing sequence of integers $n_{k}, k=1,2, \ldots$, there exist functions

$$
u, v \text { and } v_{\alpha}, 1 \leqslant|\alpha| \leqslant 2
$$


in $L^{2}(Q)$ such that

$$
\begin{aligned}
& \widetilde{u_{n_{k}}} \rightarrow u \text { weakly in } L^{2}(Q), k \rightarrow \infty, \\
& \widetilde{\partial_{t} u_{n_{k}}} \rightarrow v \text { weakly in } L^{2}(Q), k \rightarrow \infty, \\
& \widetilde{\partial^{\alpha} u_{n_{k}}} \rightarrow v_{\alpha} \text { weakly in } L^{2}(Q), k \rightarrow \infty,
\end{aligned}
$$

$1 \leqslant|\alpha| \leqslant 2$. Clearly,

$$
v=\partial_{t} u, v_{\alpha}=\partial^{\alpha} u, 1 \leqslant|\alpha| \leqslant 2
$$

in the sense of distributions in $Q$, then in $L^{2}(Q)$. So, $u \in \mathcal{H}^{1,2}(Q)$ and

$$
\partial_{t} u-\sum_{i=1}^{2} a_{i}\left(t, x_{1}, x_{2}\right) \partial_{i i} u+\sum_{i=1}^{2} b_{i}\left(t, x_{1}, x_{2}\right) \partial_{i} u+c\left(t, x_{1}, x_{2}\right) u=f \text { in } Q
$$

On the other hand, the solution $u$ satisfies the boundary conditions $\left.u\right|_{\partial Q \backslash \Sigma_{T}}=0$, since

$$
\forall n \in \mathbb{N}^{*},\left.u\right|_{Q_{n}}=u_{n}
$$

This proves the existence of a solution to Problem (1.1). Notice that we have the estimate

$$
\|u\|_{\mathcal{H}^{1.2}(Q)} \leqslant K\|f\|_{L^{2}(Q)},
$$

which implies the uniqueness of the solution.

Remark 2.2. If $\varphi_{1}(0)<\varphi_{2}(0)$ and $\varphi_{1}(T)=\varphi_{2}(T)$, then the result given in Theorem 1.1 holds true under the assumption

$$
\varphi_{k}^{\prime}(t) \varphi(t) \rightarrow 0 \quad \text { as } t \rightarrow T, \quad k=1,2
$$

instead of hypothesis (1.2).

The authors want to thank the anonymous referee for a careful reading of the manuscript and for his/her helpful suggestions.

\section{References}

[1] B.-E.Ainseba, J.-P. Kernevez, R.Luce, Application des sentinelles a l'identification des pollutions dans une rivière, Mathematical modelling and Numerical analyses, 3(1994), no. 28, $297-312$.

[2] Yu.A.Alkhutov, $L_{p}$-Estimates of solutions of the Dirichlet problem for the heat equation in a ball, J. Math. Sc., 142(2007), no. 3, 2021-2032.

[3] V.N.Aref'ev, L.A.Bagirov, Asymptotic behavior of solutions to the Dirichlet problem for parabolic equations in domains with singularities, Mathematical Notes, 5(1996), no. 1, 10-17.

[4] E.A.Baderko, M.F.Cherepova, The first boundary value problem for parabolic systems in plane domains with nonsmooth lateral boundaries, Doklady Mathematics, 90(2014), no. 2, $573-575$.

[5] R.M.Brown, W.Hu, G.M.Lieberman, Weak solutions of parabolic equations in noncylindrical domains, Proc. Amer. Math. Soc., 125(1997), 1785-1792. 
[6] P.Cannarsa, G.Da Prato, J.-P.Zolèsio, Evolution equations in non-cylindrical domains, Atti Accad. Naz-lincei cl. Sci. Fis Mat. Natur., 88(1990) no. 8, 73-77.

[7] M.Dauge, Elliptic Boundary Value Problems on Corner Domains, Springer, Berlin, 1988.

[8] S.P.Degtyarev, The solvability of the first initial-boundary problem for parabolic and degenerate parabolic equations in domains with a conical point, Sbornik Math., 201(2010), no. 7, 999-1028.

[9] G.Fichera, Sulle equazioni differenziali lineari ellittico-paraboliche del secondo ordine, Atti Acc. Naz. Lincei Mem. Ser. 5, (1956), no. 8, 1-30.

[10] N.Garofalo, E.Lanconelli, Wiener's criterion for parabolic equation with variable coefficients and its consequences, Trans. Amer. Math. Soc., 308(1988), 811-836.

[11] P.Grisvard, Elliptic Problems in Non-smooth Domains, Monographs and Studies in Mathematics, 24 Pitman, Boston, MA, 1985.

[12] P.Grisvard, Singularities in Boundary Value Problems, RMA, 22 Masson, Paris, 1992.

[13] A.F.Guliyev, S.H.Ismayilova, Mixed boundary-value problem for linear second-order nondivergent parabolic equations with discontinuous coefficients, Ukrainian Mathematical Journal, 66(2015), no. 11, 1615-1638.

[14] Y.Jiongmin, Weak solutions of second order parabolic equations in noncylindrical domains, J. Partial Differential Equations, 2(1989), no. 2, 76-86.

[15] A.Kheloufi, R.Labbas, B.K.Sadallah, On the resolution of a parabolic equation in a nonregular domain of $\mathbb{R}^{3}$, Differ. Equat. Appl., 2(2010), no. 2, 251-263.

[16] A.Kheloufi, B.-K.Sadallah, On the regularity of the heat equation solution in non-cylindrical domains: two approaches, Appl. Math. Comput., 218(2011), 1623-1633.

[17] A.Kheloufi, Parabolic equations with Cauchy-Dirichlet boundary conditions in a non-regular domain of $\mathbb{R}^{N+1}$, Georgian Math. J., 21(2014), no. 2, 199-209.

[18] V.A.Kondrat'ev, Boundary problems for parabolic equations in closed regions, Am. Math. Soc. Providence. R I., (1966), 450-504.

[19] V.A.Kondrat'ev, O.A.Oleinik, Boundary-value problems for partial differential equations in nonsmooth domains, Usp. Mat. Nauk, 38(1983), no. 2, 1-66 (in Russian).

[20] V.A.Kozlov, Coefficients in the asymptotic solutions of the Cauchy boundary-value parabolic problems in domains with a conical point, Siberian Math. J, 29(1988), 222-233.

[21] V.A.Kozlov, V.G.Maz'ya, On singularities of a solution to the first boundaryvalue problem for the heat equation in domains with conical points, I, Izv. Vyssh. Uchebn. Zaved., Mat, 2(1987), 38-46 (in Russian).

[22] V.A.Kozlov, V.G.Maz'ya, On singularities of a solution to the first boundaryvalue problem for the heat equation in domains with conical points II, Izv. Vyssh. Uchebn. Zaved., Mat., 3(1987), 37-44 (in Russian).

[23] R.Labbas, M.Moussaoui, On the resolution of the heat equation with discontinuous coefficients, Semigroup Forum, 60(2000), 187-201.

[24] O.A.Ladyzhenskaya, V.A.Solonnikov, N.N.Ural'tseva, Linear and Quasi-Linear Equations of Parabolic Type, A.M.S., Providence, Rhode Island, 1968. 
[25] J.L.Lions, Sur les problèmes mixtes pour certains systèmes parboliques dans des ouverts non cylindriques, (1957), Ann. Inst. Fourier, 143-182.

[26] J.L.Lions, E.Magenes, Problèmes aux Limites Non Homogènes et Applications, 1, 2 Dunod, Paris, 1968.

[27] G.Lumer, R.Schnaubelt, Time-dependent parabolic problems on non-cylindrical domains with inhomogeneous boundary conditions, J. evol. equ., 1(2001), 291-309.

[28] A.Maugeri, D.K.Palagachev, L.G.Softova, Elliptic and parabolic equations with discontinuous coefficients, Vol. 109 of Mathematical Research, Wiley-VCH Verlag Berlin GmbH, Berlin, 2000.

[29] O.A.Oleinik, A problem of Fichera, Doklady Akad. Nauk SSSR, 157(1964), 1297-1301 (in Russian).

[30] B.K.Sadallah, Etude d'un problème 2m-parabolique dans des domaines plan non rectangulaires, Boll. Un. Mat. Ital., 2-B(1983), no. 5, 51-112.

[31] M.-E.Stoeckel, R.Mose, P.Ackerer, Application of the sentinel method in a groundwater transport model, Transactions on Ecology and the environment, 17(1998), 673-680

[32] M.Taniguchi, Initial boundary value problem for the wave equation in a domain with a corner, Tokyo J. Math, 16(1993), no. 1, 61-98.

\title{
О линейном параболическом уравнении второго порядка с переменными коэффициентами в нерегулярной области $\mathbb{R}^{3}$
}

\author{
Ферроди Булкоан \\ Факультет естественных наук и жизни \\ Университет Беджайа, 6000, Беджайа \\ Алжир \\ Арезки Келуфи \\ Университет Беджайа, 6000, Беджайа \\ Алжир
}

Факультет технологии, лаб. прикладной математики

Настоящая работа посвящена изучению следующего параболического уравнения с переменными коэфбициентами в недивергентной форме:

$$
\partial_{t} u-\sum_{i=1}^{2} a_{i}\left(t, x_{1}, x_{2}\right) \partial_{i i} u+\sum_{i=1}^{2} b_{i}\left(t, x_{1}, x_{2}\right) \partial_{i} u+c\left(t, x_{1}, x_{2}\right) u=f\left(t, x_{1}, x_{2}\right),
$$

с учетом граничных условий Коши-Дирихле. Задача задана в нерегулярной области вида

$$
\left.Q=\left\{\left(t, x_{1}\right) \in \mathbb{R}^{2}: 0<t<T, \varphi_{1}(t)<x_{1}<\varphi_{2}(t)\right\} \times\right] 0, b[,
$$

где $\varphi_{k}, \quad k=1,2$ являются гладкими функииями. Одной из основных задач этой работв служит то, что область может бытъ нерегулярной, например, допускается особый случай, когда $\varphi_{1}$ совпадает с $\varphi_{2}$ при $t=0$. Анализ проводится в рамках анизотропных пространств Соболева с использованием метода декомпозиции областей. Эта работа является обобщением случая постоянных коэффициентов, изучаемого в [15].

Ключевые слова: параболические уравнения, нерегулярные области, переменные коэфбициенты, анизотропные пространства Соболева. 